\title{
Quantum Loop Topography for Machine Learning
}

\author{
Yi Zhang ${ }^{*}$ and Eun-Ah Kim ${ }^{\dagger}$ \\ Department of Physics, Cornell University, Ithaca, New York 14853, USA \\ and Kavli Institute for Theoretical Physics, University of California, Santa Barbara, California 93106, USA
}

(Received 15 November 2016; revised manuscript received 13 February 2017; published 22 May 2017)

\begin{abstract}
Despite rapidly growing interest in harnessing machine learning in the study of quantum many-body systems, training neural networks to identify quantum phases is a nontrivial challenge. The key challenge is in efficiently extracting essential information from the many-body Hamiltonian or wave function and turning the information into an image that can be fed into a neural network. When targeting topological phases, this task becomes particularly challenging as topological phases are defined in terms of nonlocal properties. Here, we introduce quantum loop topography (QLT): a procedure of constructing a multidimensional image from the "sample" Hamiltonian or wave function by evaluating two-point operators that form loops at independent Monte Carlo steps. The loop configuration is guided by the characteristic response for defining the phase, which is Hall conductivity for the cases at hand. Feeding QLT to a fully connected neural network with a single hidden layer, we demonstrate that the architecture can be effectively trained to distinguish the Chern insulator and the fractional Chern insulator from trivial insulators with high fidelity. In addition to establishing the first case of obtaining a phase diagram with a topological quantum phase transition with machine learning, the perspective of bridging traditional condensed matter theory with machine learning will be broadly valuable.
\end{abstract}

DOI: 10.1103/PhysRevLett.118.216401

Introduction.-Machine learning techniques have been enabling neural networks to recognize and interpret big data sets of images and speeches [1]. Through supervised training with a large set of data, neural networks "learn" to recognize key features of a universal class. Very recently, rapid and promising development has been made from this perspective on numerical studies of condensed matter systems, including dynamical systems [2-6] as well as classical and quantum systems undergoing phase transitions [7-13]. Also established is the theory connection to the renormalization group $[14,15]$. Exciting successes in the application of machine learning to symmetry-broken phases [7-10] may be attributed to the locality of the defining property of the target phases: the order parameter field. The snapshots of order parameter configuration form images readily fed into neural networks, well developed to recognize patterns in images.

Unfortunately, many novel states cannot be detected through a local order parameter. All topological phases are intrinsically defined in terms of nonlocal topological properties. Not only do many-body localized states of growing interest [16] fit into this category, but even a superconducting state fits in here since the superconducting order parameter explicitly breaks the particle number conservation [17]. In order for neural networks to learn and recognize such phases, we need to supply them with "images" that contain relevant nonlocal information. Clearly, information based on a single site is insufficient. One approach to the topological phase was to add complexity to the neural network architecture and use layers of convolutional filters to detect local constraints in the presence of translational symmetry, targeting a single topological phase at a time $[7,10]$. Another approach was to detect the topological edge states [13]. In addition, an ensemble of the Green's function was used to detect charge-ordered phases [9].

Here, we introduce quantum loop topography (QLT): a procedure that designs and selects the input data based on the target phases of interest guided by relevant response functions. We focus on the fermionic topological phases, but the procedure can be generalized to other situations that are not captured by purely local information as all physically meaningful states are characterized by their response functions. The subject of topological phases of matter has grown with the appeal that topological properties are nonlocal and hence, more robust [18-20]. Ironically, this attractive feature makes it difficult to detect and identify topological phases even in numerics. Importantly, detection of strongly correlated topological phases as fractional quantum Hall states [21,22], fractional Chern insulators $[23,24]$, and quantum spin liquids [25-27] requires arduous calculations of topological entanglements entropies $[28,29]$. On the other hand, quantization [21-24,30-34] is a natural theme of topological states, and one may wonder whether there is an intelligent way to detect them due to the discreteness in defining properties. In this Letter, we demonstrate that QLT enables even a rather simple architecture consisting of a fully connected neural network with a single hidden layer to recognize the Chern insulator and the fractional Chern insulator states and rapidly produce a phase diagram containing topological quantum phase transition (TQPT). We then discuss insights into the 


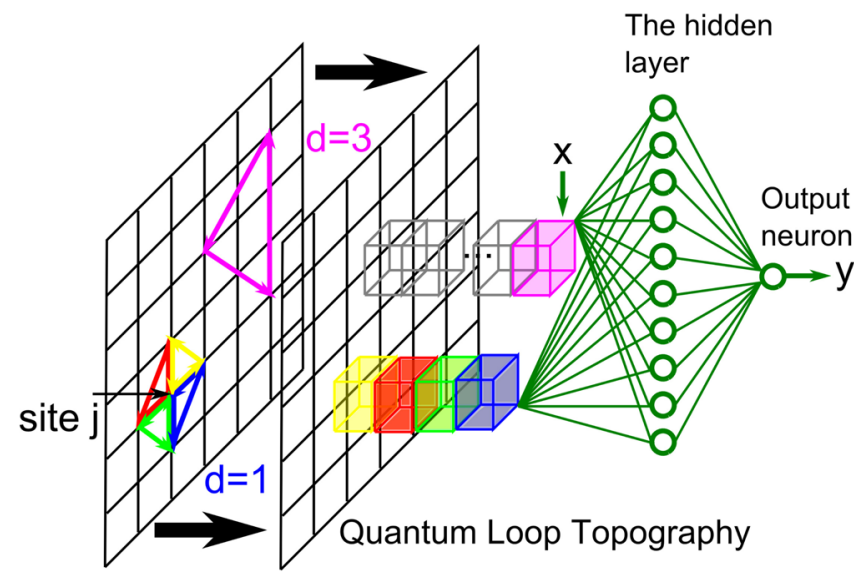

FIG. 1. Schematic illustration of our machine learning algorithm that consists of QLT and a neural network architecture. QLT for each site $j$ consists of 4 loops of linear dimension $d=1$. One loop of $d=3$ is also shown for illustration. QLT of $d \leq d_{c}$ form a $D\left(d_{c}\right)$-dimensional vector for each site $j$, e.g., $D(1)=4$ on a square lattice.

effectiveness of QLT and future directions based on its versatility.

Quantum loop topography and our algorithm.-The procedure we dubbed QLT constructs an input image from a given Hamiltonian or many-body wave function that contains a minimal but sufficient amount of nonlocal information. The response function that characterizes the phase of interest determines the geometry of the loop objects that enter QLT. But instead of a brute-force evaluation of the response functions, we use QLT obtained from instances of Monte Carlo calculations to train and utilize a network.

For Chern insulators, the relevant response function is the Hall conductivity. Interestingly, Kitaev [35] pointed out that

$$
\sigma_{x y}=\frac{e^{2}}{h} \frac{1}{N} \sum 4 \pi i P_{j k} P_{k l} P_{l j} S_{\triangle j k l}
$$

for free fermion systems [36], where $P_{i j} \equiv\left\langle c_{i}^{\dagger} c_{j}\right\rangle$ is the equal-time two-point correlation function between sites $i$ and $j, S_{\triangle j k l}$ is the signed area of triangle $j k l$, and $N$ is the total number of sites. Taking hints from Eq. (1), we use triangular loops to define QLT for Chern insulators. But instead of the full expectation value for two-point correlation functions in Eq. (1), which are costly to evaluate (requiring many Monte Carlo steps walking down the Markov chain), we evaluate the bilinear operator with a single Monte Carlo sample $\alpha$, defining $\left.\tilde{P}_{j k}\right|_{\alpha} \equiv\left\langle c_{j}^{\dagger} c_{k}\right\rangle_{\alpha}$. Further, we note that smaller triangles dominate in a gapped system and keep the loops of linear dimension $d \leq d_{c}$, where $d_{c}$ is the cutoff.

Now, we define QLT to be a quasi-two-dimensional "image" of the $D\left(d_{c}\right)$-dimensional vector of complex numbers assigned to each lattice site $j$. Each entry of this vector is associated with a distinct triangle cornered at site $j$, which defines a chained product

$$
\left.\left.\left.\tilde{P}_{j k}\right|_{\alpha} \tilde{P}_{k l}\right|_{\beta} \tilde{P}_{l j}\right|_{\gamma},
$$

where $k$ and $l$ are two other sites of the particular triangle, and $\tilde{P}$ 's are evaluated at three independent Monte Carlo steps without averaging over Markov chain. $D\left(d_{c}\right)$ is the total number of triangles, with $d \leq d_{c}$ assigned to each site (see Fig. 1). This way, QLT can be systematically expanded to include longer-ranged correlations by increasing the cutoff $d_{c}$ until convergence.

By construction, QLT is quite versatile. Firstly, QLT can be obtained for and mapped between different lattice geometry, which only enters through $D\left(d_{c}\right)$. Secondly, the entire procedure takes place in a real space without the need for diagonalization or flux insertion and does not depend on translational invariance. Hence, QLT should naturally accommodate heterogeneity, disorder, and interaction. Finally, it is clear that the strategy underlying QLT construction that we have laid out can be generalized to other novel phases, such as $\mathbb{Z}_{2}$ topological order, quantum spin Hall insulator, or superconductivity [38]. In the rest of this Letter, we use variational Monte Carlo (VMC) calculations, without loss of generality, to build QLT by sampling the many-body ground states of interest at randomly selected Monte Carlo steps (see Supplemental Material [37]).

Once QLT is obtained for a given model, we feed it to a neural network (Fig. 1). For this, we design a feed-forward fully connected neural network with only one hidden layer consisting of $n=10$ sigmoid neurons. The network takes QLT as an input $x$, and each neuron processes the input through independent weights and biases $w x+b$. After the sigmoid function, the outcome is fed forward to be processed by the output neuron. The final output $y$ corresponds to the neural network's judgement whether the input QLT is topological. We use cross entropy as the cost function with L2 regularization to avoid overtraining and a mini-batch size of 10 [1]. For the rest of this Letter, we use randomly mixed 20000 data samples within the VMC Metropolis of the topological and trivial phases as the training group. We reserve a separate group of 4000 data samples (also half trivial and half topological) for validation purposes, including learning speed control and termination [1]. Once the machine learning is successful, the trained neural network can rapidly process QLTs from different parts of the phase space to yield a phase diagram. In order to establish a level of confidence on the trained network's assessment of whether the system is topological or not, we process 2000 QLTs at each point and take the ratio $p$ of the "topological" output, i.e., $y>0.5$. When $p$ is close to 1 for topological phase and 0 for trivial phase, it indicates that even a single QLT can reliably land a trustworthy detection.

Topological quantum phase transition in a free fermion model.-We first apply the QLT-based machine learning to the TQPT between the trivial insulator and the Chern insulator. Consider the following tight-binding model on a square lattice: 


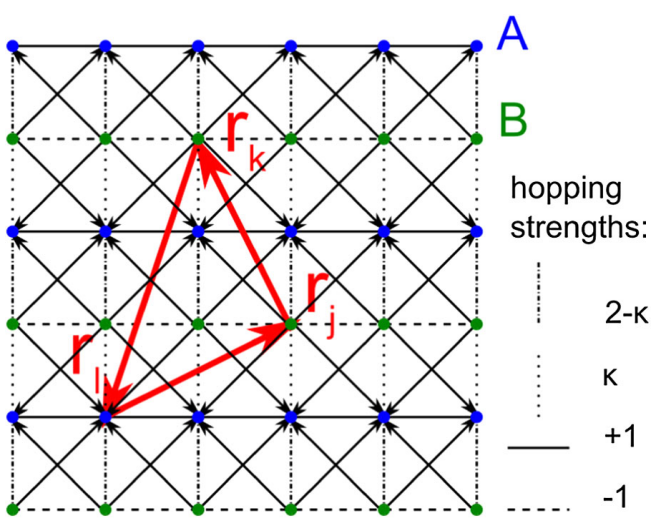

FIG. 2. Model illustration of Eq. (3). The unit cell consists of two sublattice sites, $A$ and $B$. Hopping strengths are different for horizontal and vertical bonds and staggered. The diagonal hopping is $i \kappa(-i \kappa)$ along (against) the arrow. The red arrows denote a triangle that defines the operators of our QLT.

$$
\begin{aligned}
H(\kappa)= & \sum_{\vec{r}}(-1)^{y} c_{\vec{r}+\hat{x}}^{\dagger} c_{\vec{r}}+\left[1+(-1)^{y}(1-\kappa)\right] c_{\vec{r}+\hat{y}}^{\dagger} c_{\vec{r}} \\
& +(-1)^{y} \frac{i \kappa}{2}\left[c_{\vec{r}+\hat{x}+\hat{y}}^{\dagger} c_{\vec{r}}+c_{\vec{r}+\hat{x}-\hat{y}}^{\dagger} c_{\vec{r}}\right]+\text { H.c. }
\end{aligned}
$$

where $\vec{r}=(x, y)$ (see Fig. 2), and $0 \leq \kappa \leq 1$ is a tuning parameter. The $\kappa=1$ limit is the $\pi$-flux square lattice model for a Chern insulator with Chern number $C=1$ [31], while the $\kappa=0$ limit amounts to decoupled two-leg ladders. $H(\kappa)$ interpolates between the Chern insulator and the trivial insulator, with a TQPT at $\kappa=0.5$. To see this, Fourier transform the Hamiltonian to momentum space

$$
\begin{aligned}
H(\kappa)= & \sum_{k}\left[2 \cos k_{y}+2 i \sin k_{y}\left(1-\kappa+\kappa \sin k_{x}\right)\right] c_{k, A}^{\dagger} c_{k, B} \\
& +2 \cos k_{x}\left(c_{k, A}^{\dagger} c_{k, A}-c_{k, B}^{\dagger} c_{k, B}\right)+\text { H.c. }
\end{aligned}
$$

where $A$ and $B$ label the two sublattices, and note that in this two-band model with two Dirac points at $( \pm \pi / 2, \pi / 2)$, one of the Dirac masses changes sign across $\kappa=0.5$.

Our complete knowledge of the phase diagram makes Eq. (3) an ideal testing ground for our algorithm. Hence, we implement supervised machine learning using two deepin-phase models of $\kappa=1.0$ (Chern insulator) and $\kappa=0.1$ (trivial insulator) for training [39]. The system size is $12 \times 12$ lattice spacings unless noted otherwise. First, we establish that indeed, single-site-based inputs of the fermion occupation configurations $n(\vec{r})=c_{\vec{r}}^{\dagger} c_{\vec{r}}$ fail to transmit the topological information to the neural network, as we expected. With $n(\vec{r})$ as inputs, the learning is inefficient, signaled by high yields in the cost function [1]. Moreover, as shown in Fig. 3, even after a long period of training, the neural network keeps incorrectly judging the system to be a trivial insulator for all values of $\kappa$, except for $\kappa=1.0$, where the result returns $>80 \%$ "nontrivial."

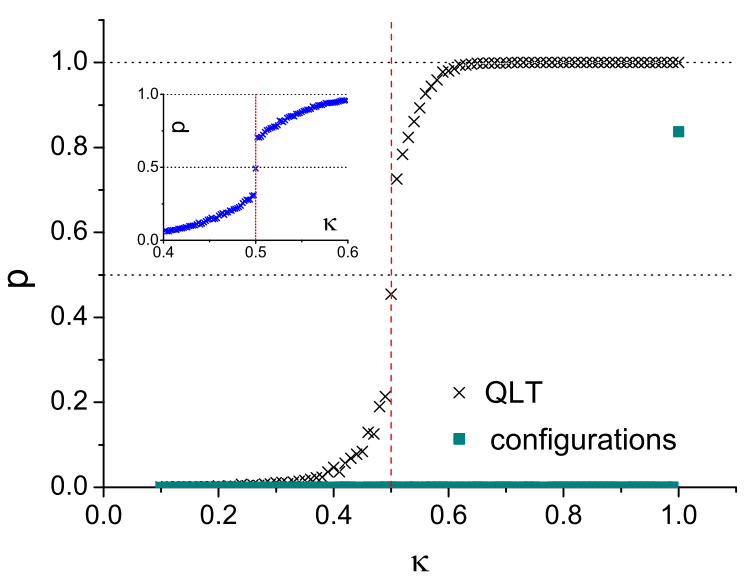

FIG. 3. The ratio $p$ of the "topological" response from the neural network on the model in Eq. (3) over the parameter region $\kappa \in[0.1,1,0]$. The neural network is trained, with $\kappa=0.1$ for $y=0$, and $\kappa=1$ for $y=1$. The green square symbols represent the results using fermion occupation configurations as an input data. The red dashed line marks the expected topological phase transition at $\kappa=0.5$. The inset: an enlarged view over the critical region $0.4 \leq \kappa \leq 0.6 . d_{c}=2$ for all.

This indicates that the neural network unfortunately does not pick up the universal features about the topological phase, but rather memorizes the details of the specific model at $\kappa=1.0$.

The contrast in the results based on QLT input is striking. Figure 3 shows that the trained network's assessment achieves $>99.9 \%$ accuracy deep in either the topological phase or the trivial phase, even with $d_{c}=2$. Moreover, even though we have provided the training group with only large-gap models in both the topological and trivial phases focusing on identifying phases [37], we find a nonanalytical behavior in $p$ as a function of $\kappa$ at the critical point (see Fig. 3 inset).

Generalizations. - Next, we consider a fractional Chern insulator (FCI) as an example of a strongly correlated topological phase. Here, the $\nu=1 / 3 \mathrm{FCI}$ is represented by a VMC wave function that is the free fermion wave function of the model in Eq. (3) raised to the third power [40]. Surprisingly, the neural network trained on the noninteracting parent Chern insulator already serves as a "poor man's network" (see the inset of Fig. 4). This network recognizes that the FCI phase is distinct from the parent Chern insulator, and hence, it only gives $p \sim 0.01$ "nontrivial" response for the FCI phase. Nevertheless, it also notices that FCI is a topologically distinct state from the trivial insulator since $p \sim 0.01$ is large enough to exclude statistical error. Once trained with the FCI wave function at two reference points, $\kappa=0.1$ for trivial and $\kappa=1.0$ for FCI, the network once again detects the FCI phase with high accuracy [see Fig. 4].

Remarkably, the network automatically recognizes topological degeneracy. Even if we train the network with only one of the degenerate ground states (GS\#1 in Fig. 4) in the 


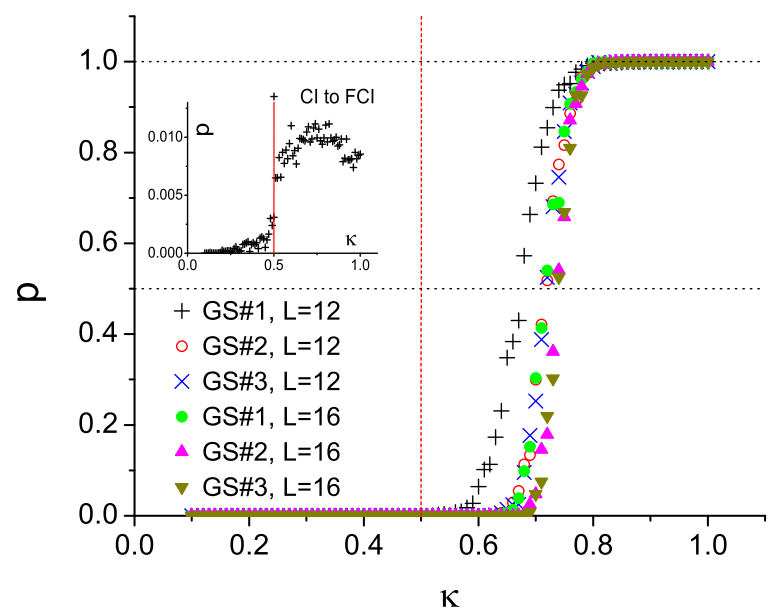

FIG. 4. Application to a $\nu=1 / 3$ FCI. The topological phase transition in the parent Chern insulator at $\kappa=0.5$ is marked by a vertical red dashed line. The inset shows the results using a neural network trained with the parent free fermion model, where $p$ is calculated over 20000 samples for each $\kappa$ to reduce statistical error. The main panel shows the results using FCI wave functions for both training ( $\kappa=0.1$ for trivial and $\kappa=1.0$ for the FCI, first ground state only) and testing (all three degenerate ground states, see Supplemental Material [37]). $L=16$ data are shown in addition to $L=12$ to help attribute the differences between $\kappa_{c}$ of the topological phase transitions to the finite-size effect. $d_{c}=2$ for all.

topological phase, it correctly assesses the topological nature of the two other ground states that are related to GS\#1 by flux threading. The network also detects a TQPT at $0.67 \leq \kappa_{c} \leq 0.77$. The uncertainty in the critical value $\kappa_{c}$ is a finite-size effect on the quasidegeneracy as is clear from the convergence upon increasing the system size. The fact that $\kappa_{c}>0.5$ when the single-particle gap closes at $\kappa=0.5$ could raise concern in light of the findings on singleparticle Green's-function-based approaches [41,42]. We note that the single-particle gap is a prerequisite for the VMC wave function to represent a topological phase since only then partons may be integrated out; hence, the shift of $\kappa_{c}>0.5$ is expected. Nevertheless, the result is the first report of the TQPT providing the target and calls for further study on the critical point using independent measures such as the many-body gap or the more established yet costly entanglement-entropy-based approaches [28,29,40,43,44].

Finally, we demonstrate that we can train the network to learn the topological protection of the topological phases and show indifference to the microscopic details, such as lattice structure or impurities. The key to successful machine learning, this celebrated feature is the diversity of the training inputs. Without diverse inputs, the network looks for features that are specific to its training set. For instance, the network trained only with square lattice cannot recognize the topological phase in the honeycomb lattice. But, if we provide diverse input taken from both the square lattice and the honeycomb lattice systems, the

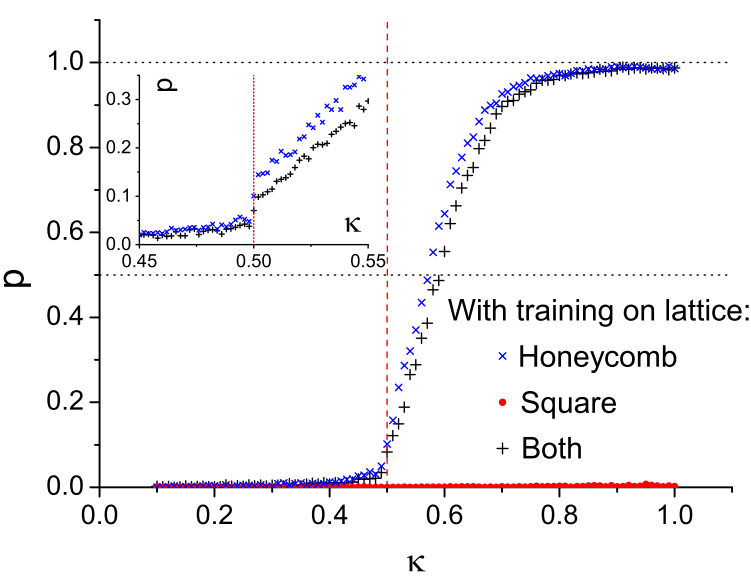

FIG. 5. The ratio $p$ of a "topologically nontrivial" response from the neural networks for the honeycomb lattice model (Supplemental Material [37]) over the parameter region $\kappa \in[0.1,1,0]$. The topological phase transition is at $\kappa=0.5$ (vertical red dashed line). The neural networks are trained using the Chern insulators and trivial insulators only on the honeycomb lattice, only on the square lattice, and on both. The inset zooms into the critical region $0.4 \leq \kappa \leq 0.6 . d_{c}=2$ for all.

network can be trained to recognize topological phases on both lattices with little penalty on accuracy (see Fig. 5). We also note that the network recognizes the difference between different Chern numbers (e.g., $C=-1$ vs $C=1$ ) as well as higher Chern numbers (e.g., $C=2$ ).

Conclusion.-In summary, we have successfully implemented supervised machine learning for topological phases by introducing QLT as an interface between the traditional concept of response theory and a simple neural network.

Three major strengths of our QLT-based machine learning approaches are 1) efficiency, 2) accuracy, and 3) versatility. Firstly, the network can be trained with quasitwo-dimensional QLT in gapped phases. Furthermore, since QLT bypasses the time-consuming process of averaging over Markov chains, one can quickly scan the phase space once the network is trained. Although our focus was on the phases, we demonstrated that nonanalyticity in the ratio of nontrivial responses allows us to pinpoint the phase transition. Finally, as a real-space-based formalism that does not require translational symmetry or diagonalization or flux insertion, QLT is quite versatile.

Our approach can also be applied to systems with other fillings as well as higher dimensions. The fact that QLT readily handles degenerate ground states adds to its versatility. Moreover, there is nothing restricting QLT to VMC data. It can be applied to Hamiltonian-based quantum Monte Carlo samples [41,42] as well as other representations of many-body wave functions, such as matrix product states and Projected entangled pair states (PEPS). Most importantly, the procedure of defining appropriate QLT guided by relevant response function we established here is readily expanded to other states of interest such as the 
superconducting state, quantum spin Hall insulator, and $\mathbb{Z}_{2}$ topological order [38]. Hence, our construction opens the door to applications of the machine learning to novel states of broad interest.

We thank E. Khatami, R. Melko, T. Neupert, and S. Trebst for insightful discussions. This work was supported by the DOE under Award No. DE-SC0010313. Y. Z. acknowledges support through the Bethe Postdoctoral Fellowship and E-AK acknowledges Simons Fellow in Theoretical Physics Award \#392182. The bulk of this work was done at KITP supported by Grant No. NSF PHY11-25915.

*frankzhangyi@gmail.com †eun-ah.kim@cornell.edu

[1] Michael Nielsen, Neural Networks and Deep Learning (Free Online Book, 2013) http://neuralnetworksanddeeplearning .com/.

[2] S. S. Schoenholz, E. D. Cubuk, D. M. Sussman, E. Kaxiras, and A. J. Liu, Nat. Phys. 12, 469 (2016).

[3] M. H. Amin, E. Andriyash, J. Rolfe, B. Kulchytskyy, and R. Melko, arXiv:1601.02036.

[4] G. Carleo and M. Troyer, Science 355, 602 (2017).

[5] G. Torlai and R. G. Melko, Phys. Rev. B 94, 165134 (2016).

[6] G. Torlai and R. G. Melko, arXiv:1610.04238.

[7] J. Carrasquilla and R. G. Melko, Nat. Phys. 18 (2017).

[8] L. Wang, Phys. Rev. B 94, 195105 (2016).

[9] P. Broecker, J. Carrasquilla, R. G. Melko, and S. Trebst, arXiv:1608.07848.

[10] K. Ch'ng, J. Carrasquilla, R. G. Melko, and E. Khatami, arXiv: 1609.02552

[11] J. Liu, Y. Qi, Z. Y. Meng, and L. Fu, Phys. Rev. B 95, 041101 (2017).

[12] L. Huang and L. Wang, Phys. Rev. B 95, 035105 (2017).

[13] T. Ohtsuki and T. Ohtsuki, J. Phys. Soc. Jpn. 85, 123706 (2016).

[14] C. Bény, arXiv:1301.3124.

[15] P. Mehta and D. J. Schwab, arXiv:1410.3831.

[16] R. Nandkishore and D. A. Huse, Annu. Rev. Condens. Matter Phys. 6, 15 (2015).

[17] D. J. Scalapino, S. R. White, and S. Zhang, Phys. Rev. B 47, 7995 (1993).

[18] A. Kitaev, Ann. Phys. (Amsterdam) 303, 2 (2003).

[19] S. Das Sarma, M. Freedman, and C. Nayak, Phys. Rev. Lett. 94, 166802 (2005)

[20] C. Nayak, S. H. Simon, A. Stern, M. Freedman, and S. Das Sarma, Rev. Mod. Phys. 80, 1083 (2008).
[21] R. B. Laughlin, Phys. Rev. Lett. 50, 1395 (1983).

[22] D. C. Tsui, H. L. Stormer, and A. C. Gossard, Phys. Rev. Lett. 48, 1559 (1982).

[23] T. Neupert, L. Santos, C. Chamon, and C. Mudry, Phys. Rev. Lett. 106, 236804 (2011).

[24] N. Regnault and B. A. Bernevig, Phys. Rev. X 1, 021014 (2011).

[25] V. Kalmeyer and R. B. Laughlin, Phys. Rev. Lett. 59, 2095 (1987).

[26] X.-G. Wen, Int. J. Mod. Phys. B 04, 239 (1990).

[27] L. Balents, Nature (London) 464, 199 (2010).

[28] A. Kitaev and J. Preskill, Phys. Rev. Lett. 96, 110404 (2006).

[29] M. Levin and X.-G. Wen, Phys. Rev. Lett. 96, 110405 (2006).

[30] K. v. Klitzing, G. Dorda, and M. Pepper, Phys. Rev. Lett. 45, 494 (1980).

[31] A. W. W. Ludwig, M. P. A. Fisher, R. Shankar, and G. Grinstein, Phys. Rev. B 50, 7526 (1994).

[32] F. D. M. Haldane, Phys. Rev. Lett. 61, 2015 (1988).

[33] M. Z. Hasan and C. L. Kane, Rev. Mod. Phys. 82, 3045 (2010).

[34] D. J. Thouless, M. Kohmoto, M. P. Nightingale, and M. den Nijs, Phys. Rev. Lett. 49, 405 (1982).

[35] A. Kitaev, Ann. Phys. (Amsterdam) 321, 2 (2006).

[36] Our alternative proof of Eq. (1) (see Supplemental Material [37]) builds on adiabatic continuity protected by the gap in the spectra without requiring that the system be noninteracting.

[37] See Supplemental Material at http://link.aps.org/ supplemental/10.1103/PhysRevLett.118.216401 for further discussion and details on the impact of training models and QLT cutoff $d_{c}$ on machine learning phases as well as phase transitions.

[38] Y. Zhang, R. G. Melko, and E.-A. Kim, arXiv:1705.01947.

[39] The system becomes decoupled two-leg ladders at $\kappa=0.0$, which is nongeneric for a two-dimensional insulator, and we choose to avoid such specialty.

[40] Y. Zhang, T. Grover, and A. Vishwanath, Phys. Rev. B 84, 075128 (2011).

[41] T. C. Lang, A. M. Essin, V. Gurarie, and S. Wessel, Phys. Rev. B 87, 205101 (2013).

[42] Y.-Y. He, H.-Q. Wu, Z. Y. Meng, and Z.-Y. Lu, Phys. Rev. B 93, 195164 (2016).

[43] Y. Zhang, T. Grover, A. Turner, M. Oshikawa, and A. Vishwanath, Phys. Rev. B 85, 235151 (2012).

[44] Y. Zhang, T. Grover, and A. Vishwanath, Phys. Rev. B 91, 035127 (2015). 